\title{
Kernos
}

Revue internationale et pluridisciplinaire de religion grecque antique

6 | 1993

Varia

\section{Artémis Amarysia. Des ports préférés d'Artémis : L'Euripe (Callimaque, Hymne à Artémis, 188)}

\section{Pierre Brulé}

\section{(2) OpenEdition \\ Journals}

\section{Édition électronique}

URL : http://journals.openedition.org/kernos/535

DOI : $10.4000 /$ kernos.535

ISSN : 2034-7871

\section{Éditeur}

Centre international d'étude de la religion grecque antique

\section{Édition imprimée}

Date de publication : 1 janvier 1993

Pagination : $57-65$

ISSN : 0776-3824

\section{Référence électronique}

Pierre Brulé, «Artémis Amarysia. Des ports préférés d'Artémis : L'Euripe (Callimaque, Hymne à

Artémis, 188) », Kernos [En ligne], 6 | 1993, mis en ligne le 07 avril 2011, consulté le 20 avril 2019. URL : http://journals.openedition.org/kernos/535; DOI : 10.4000/kernos.535 
Kernos, 6 (1993), p. 57-65.

\title{
ARTÉMIS AMARYSIA. \\ DES PORTS PRÉFÉRÉS D'ARTÉMIS : L'EURIPE
}

(Callimaque, Hymne à Artémis, 188)

\begin{abstract}
C'est le mythe qui m'a fait aborder pour la première fois à Brauron. J'avais comme d'autres été sensible à la grande parenté des récits attachés à Aulis, Brauron et Mounichie, mettant en scène Iphigénie, des ours(es), Artémis, un devin, un oracle, des meurtres d'animaux sacrés, et la fondation de rituels féminins. J'étais, et je suis encore, assez largement en accord avec l'analyse que W. Sale (1975) a proposée de ces récits, analyse qui affermit l'idée de leur unité d'ensemble, cela malgré l'existence de versions qu'il considère comme inconciliables. Le fait est évidemment d'autant plus frappant que les trois sites en question sont proches les uns des autres, et qu'il s'agit de trois sanctuaires d'Artémis. Il restait à W. Sale à expliquer l'unité d'ensemble en même temps que la diversité de détails des récits. C'est ainsi qu'il lui parut naturel d'imaginer qu'une version brauronienne serait allée à Aulis et à Mounichie ou qu'une version aulidienne serait venue à Brauron et Mounichie. Il optait ainsi pour une interprétation «historisante", génétique, du corpus. En un lieu avant d'autres, on aurait imaginé une histoire utilisant les ingrédients cités plus haut, aition d'un rituel féminin comportant le mime d'un animal. La variabilité des versions trouvant assez naturellement son explication dans la transformation, l'adaptation du mythe à son environnement nouveau. Un mythe a bien sûr pu vagabonder. Mais, l'hypothèse d'une communauté cultuelle définie en termes géographiques me semble meilleure. Dans la mesure où aucune version n'est susceptible d'être située chronologiquement par rapport aux deux autres, la recherche du mythe étiologique originel est un leurre. Rien ne nous est accessible dans ce domaine au-delà de la structure de ces récits. C'est donc dans une autre direction et sur d'autres terrains qu'il faut mener l'enquête.

Les croyances, les cultes ainsi que les caractéristiques matérielles des sanctuaires révèlent d'autres éléments de la cohérence du corpus; j'en ai fait des objets de l'enquête (Brulé, 1987, p. 186 sq.) : la topographie, les rituels, les vestiges architecturaux, les épiclèses divines... Cela permit d'aboutir à quelques conclusions fermes : les
\end{abstract}


mythes ne constituent pas le seul point commun entre les trois sites, mais tout un faisceau de "signes» les unissent. Pour être les mieux connus, les trois sites ne sont pas les seuls, loin de là, à répondre aux critères nouveaux ainsi définis. Et l'observation d'évidence faite pour les trois premiers vaut aussi pour les autres : une disposition géographique particulière sur la côte orientale de la Grèce. Cette découverte confortait mon opinion première : plutôt que de tenter de reconstituer une inconnaissable histoire des croyances et des rites attachés à ces sanctuaires, mieux valait se montrer attentif à leur répartition.

$\mathrm{Au}$ nombre des caractères particuliers des sanctuaires du corpus géographique je comptais : l'existence d'un adyton, d'un héroôn, une double présence de l'eau aux abords du sanctuaire : la mer et un fleuve, la pratique de sacrifices d'expiation, le rôle de la chèvre, le mime d'un animal, le caractère topographique de l'épiclèse de l'Artémis là vénérée, le culte d'Iphigénie, une certaine forme définie plus haut de mythe étiologique (tableau récapitulatif du corpus géographique, Brulé, 1987, p. 199); s'y ajoute la preuve qu'en un certain nombre de ces sanctuaires on utilisait les mêmes vases rituels, ceux que l'on a coutume maintenant d'appeler les cratérisques brauroniens (p. 251). Parmi les sites qui me semblaient devoir légitimement faire partie de l'ensemble certains fournissaient, par manque de renseignements, peu d'intersections dans le tableau final.

Deux épiclèses d'Artémis avaient en particulier attiré mon attention. Une Artémis Amarynthia et une Amarysia. Le scholiaste au vers 875 des Oiseaux d'Aristophane commentant une invention ornithologique du poète : une Artémis Akalanthis (chardonneret), signale qu'elle fait référence à une Kolainis qu'Euphronios localise en Eubée à Amarynthos, parce que c'est à elle qu'Agamemnon sacrifia un bélier taré (citant Callimaque au passage [Pfeiffer 200]). Selon le scholiaste, cette Artémis Kolainis qu'Euphronios place à Amarynthos, existe aussi à Myrrhinonte sur la côte orientale de l'Attique, à proximité de Brauron. Puis il prend le commentaire à son compte pour ajouter :

les gens de Myrrhinonte appellent cette Artémis Kolainis comme ceux du Pirée Mounichia et les Philaïdes Braurônia.

Cela me semble signifier que le scholiaste interprète l'épiclèse comme je le fais : il lui donne une valeur topographique. Nous voilà donc avec deux Artémis Kolainis, l'une eubéenne et l'autre attique. 
Constatant que les habitants du petit dème d'Athmonte, au nord-est d'Athènes, honorent une Artémis Amarysia, Pausanias, déçu de ce que les «guides ne savent rien de précis», donne sa propre explication :

Il y a en Eubée un village d'Amarynthos, dont les habitants ont aussi un culte d'Amarysia; or les Athéniens célèbrent une fête d'Amarysia qui n'a pas moins d'éclat que celle de l'Eubée : c'est de là, à mon avis, qu'est venu chez les Athmonéens le nom de la déesse (I, 34, 4-5).

Je persiste à penser que le raisonnement de Pausanias a de bonnes chances d'être juste : le phénomène qu'il décrit est celui de l'épiclèse topographique déplacée que j'ai rencontré plusieurs fois au cours de cette étude. Mais tout cela est bien confus. Amarynthos a-t-elle connu deux Artémis "maritime(s)": la Kolainis et l'Amarysia, la première ayant colonisé Myrrhinonte et la seconde Athmonte? Ou n'y existait-il "primitivement" que l'Amarysia? Avec cette conséquence que la Kolainis amarynthienne connue d'Euphronios serait une épiclèse déplacée en provenance de Myrrhinonte ? La seconde hypothèse semble la plus probable : Amarysia, identique à l'Amarynthia (déjà a-ma-ru$t a$ en mycénien), est bien l'épiclèse topographique correspondant à Amarynthos. Kolainis, en revanche, ne me semble pas renvoyer à un toponyme identifiable. On verra par ailleurs tout à l'heure qu'il est une autre candidate parmi les épiclèses topographiques.

Mais tout cela était un peu maigre, et l'on comprendra ma curiosité en découvrant dans la dernière livraison de Kernos le compte rendu de la fouille d'un dépôt votif sur les lieux de ce que l'on suppose avoir été le sanctuaire d'Artémis Amarysia par E. Sapouna-Sakellaraki.

Que savions-nous jusqu'alors du culte ? Par Strabon on pouvait évoquer les splendeurs d'une procession avec soldats en armes, cavaliers et chars $(\mathrm{X}, 1,10)$. Une inscription de l'époque classique mentionne des concours en l'honneur d'Artémis Amarynthia comportant une compétition musicale, des danses, particulièrement la pyrrhique ( $A E, 1902$, p. 99; $I G$, XII 9, 236; Ringwood, 1927, p. 385 sq.). Ce sont des fouilles clandestines qui ont permis la mise au jour d'un dépôt important (plus de 3 ares) à $0,60 \mathrm{~m}$ de profondeur sur une épaisseur de $80 \mathrm{~cm}$ environ. Il fut en usage du début de l'époque archaïque jusqu'à l'époque byzantine. Le contenu de ce dépôt éveille de nombreux souvenirs brauroniens.

Il ne s'agit pas d'une publication systématique mais d'une rapide typologie des objets du dépôt. Je suis l'ordre proposé par E. SapounaSakellaraki (je renvoie une fois pour toutes à son texte pour les justifications). 
1. Des fibules de "type insulaire» ainsi que les bagues, objets à usage privé féminins rappellent évidemment les dons votifs brauroniens. L'intérêt, c'est aussi leur date : VIIIe-VIIe, ainsi que leur type morphologique.

2. Communs avec Brauron aussi ces fragments de couvercles de pyxides en marbre blanc.

3. Des objets de verre bleu "de type phénicien» en petit nombre; la fouille de Brauron a donné des verres semblables.

4. «Bon nombre» de pesons de fuseau - poids de tisserand de plusieurs types. On en connaissait déjà l'existence par une trouvaille fortuite : un poids avec anthema et une dédicace à Artémis.

5. On aimerait pouvoir mieux connaître la céramique "abondamment représentée». "Les vases les plus anciens remontent à la fin de la période géométrique et archaïque ancienne». Artémis veuille qu'on attende moins longtemps que pour la céramique de Brauron ! À la lecture du compte rendu, deux types de vases me semblent devoir attirer l'attention : a) "nombre" de calices à figures noires des petits maîtres avec des représentations de ce qui semble être le contenu des cérémonies auxquelles l'inscription fait allusion : scènes dionysiaques, chars, athlétisme... b) des vases avec de petits dessins de formes variées mais évoquant de petites corbeilles. "Il faut souligner que des vases de ce type ont été découverts dans le Thesmophorion d'Érétrie, ce qui prouve leur caractère cultuel».

6. Parmi les reliefs en argile, un «fragment de plaque avec une tête portant une coiffure conique, comme celle de l'Artémis Bendis; derrière la tête, un relief représente un arbre, peut-être un palmier".

7. Parmi les statuettes de femme, dont on a l'impression qu'elles sont nombreuses, il faut sans doute retenir cette série d'Artémis assise sur un trône, tenant «un petit lion, un cerf, une fleur ou un petit enfant».

8. «Une multitude (?) de statuettes... représentant des enfants filles et garçons». On aimerait avoir une idée de la proportion des sexes. «On trouve parmi les statuettes des "crouching boys", des enfants consacrés dans les sanctuaires». La présence de ces enfants accroupis excite évidemment notre curiosité. E. Sapouna-Sakellaraki ne distingue ensuite pas moins de 14 types de statuettes. Doivent, me semble-t-il, retenir particulièrement l'attention le type de filles portant un kanistron; celui des filles en manteau avec tambourin, sac, grappe de raisin; enfants tenant une couronne ou la portant au cou. 
On ajoutera aussi, pêle-mêle : des couples : la Kourotrophe et le petit enfant debout; de nombreux animaux : cerf, lion, taureau, agneau, renard, coq, perdrix, cheval, colombe, cigale, abeille, tortue, porc, bélier, chien, serpent. L'association enfant-animal est fréquente : avec le chien, avec le coq et assis avec un oiseau. "Enfin, on trouve de nombreuses statuettes d'acteurs avec des masques provenant de la Nouvelle Comédie qui commencent vers 330 . Le type le plus courant est celui du Paposilène..."

L'auteur conclut, sans risque, que nous avons affaire à un dépôt qui "peut être associé au culte d'Artémis, et, de ce fait, à l'Artémis d'Amarynthos".

Un certain nombre d'éléments de cette présentation justifient la notion de corpus géographique que j'avais proposée. On ne peut, je crois, qu'être sensible aux nombreux parallèles qui doivent être tracés entre Amarynthos et Brauron (et subsidiairement avec d'autres sanctuaires maritimes).

Un des traits les plus apparents de cette nouvelle Artémis ce sont ses rapports avec le monde féminin.

J'attache la plus grande importance dans ce contexte à l'offrande des pesons de tissage. Le fait peut être considéré en soi comme banal. Combien de pesons, dans combien de sanctuaires? Il ne l'est pas s'agissant d'Artémis qui ne jouit pas des mêmes vertus de tisseuses qu'une Athéna, à la fois en définition et en extension. Ni aussi réputée, ni dans une aire géographique aussi vaste. Le phénomène est connu à Brauron avec les fameuses offrandes textiles, et compte tenu de ce qu'il s'agit là d'une singularité de la Brauronia, cet usage désigne l'Amarysia comme sa très proche parente. De la même façon les objets féminins : fibules et pyxides de marbre apparaissent dans la très ample moisson d'objets en rapport avec la vie privée des femmes découverte à Brauron (toujours inédite).

En relation avec la vie des femmes aussi l'importance des représentations en rapport avec la génération. J'en veux pour preuve le nombre et la diversité des figures de kourotrophes. Iphigénie est-elle présente ici comme à Brauron, à Aulis, et dans d'autres sanctuaires du corpus ? Dans ce contexte, la trace d'une présence d'Agamemnon à Amarynthos, signalée plus haut, est troublante. S'agissant, par ailleurs, des puissances divines protectrices de l'accouchement et de la petite enfance, il faut signaler la présence, vérifiée par une inscription, d'une Artémis Eileithyia en Eubée (IG, XII 9, 924). La valence identique 
d'Eileithyia et d'Iphigénie dans leurs rapports à Artémis rend possible une permutation entre les deux épiclèses.

La découverte de cratérisques rituels à Mounichie et à Halai avait déjà offert une vérification de la pertinence de la mise en série des sanctuaires maritimes d'Artémis. La typologie présentée par E. Sapouna-Sakellaraki met en évidence une classe de petits vases en forme de corbeille. Le fait qu'on en ait découvert de ce même type au Thesmophrion d'Érétrie indique qu'ils sont en rapport avec un gestuel féminin de caractère rituel. Malheureusement aniconiques, ils ne nous renseignent pas comme ceux du continent sur le détail des rituels.

Dans la perspective où je me place, il serait important de vérifier la présence du palmier sur le fragment de plaque (6). La céramique brauronienne lui fait une place importante, dans un contexte cultuel, et les fouilles de Mounichie en donnent elles aussi des représentations : plaque d'argile et à l'arrière de l'autel des cérémonies nocturnes des ourses sur un cratérisque (Palaiokrassa, 1983, pin. 31 et 44). On ne peut manquer d'être sensible à l'unité de la zone géographique couverte par le palmier délien.

Il n'est pas jusqu'aux énigmes qui, à Amarynthos, rappellent celles de Brauron. Ainsi la présence de Dionysos (sur la céramique classique à figures noires). La question était déjà épineuse à Brauron, il vaut mieux, ici, attendre de mieux connaître le détail des découvertes.

L'intérêt de la constitution d'un corpus dans une telle enquête c'est que chaque élément de l'ensemble peut aider à interpréter les données fournies par les autres. Une des crux de Brauron c'est une lacune des sources textuelles en regard des sources archéologiques : les premières ne connaissent que des fillettes, les secondes, c'est-à-dire quelques cratérisques et beaucoup de statues, montrent aussi des garçons. Or les aitia ne sont d'aucune utilité pour comprendre la présence des seconds. D'où cet embarras, sensible au fait que les études sur Brauron passent régulièrement sous silence cette présence des garcons que remarque tout visiteur du Musée. Et voilà que le même parallèle peut être tracé à Amarynthos : une multitude de statuettes d'enfants, filles et garçons.

L'impatience d'en savoir plus, beaucoup plus (ah! l'âge des fillettes et des garçonnets...), sur les résultats de la fouille, ne nous empêche pas de tirer tout de suite quelques conclusions de ce relevé rapide.

Nous nous trouvons en présence d'un complexe cultuel d'une grande richesse. Il est certain que les croyances et les rituels ne se présentent pas ici selon le modèle : un dieu/héros, une croyance, une pratique. Comme en face, à Brauron (et ailleurs), la divinité topique est multi- 
fonctionnelle; on la voit et on la devine kourotrophe, protectrice des concours, protectrice des activités féminines - et des femmes -, Potnia thèrôn. Et je conclurai pour l'instant en disant que le sanctuaire d'Artémis Amarysia appartient sans ambiguïté au corpus des sanctuaires maritimes d'Artémis, tout en possédant quelques caractéristiques propres

Stace évoque les liens qui unissent Artémis aux littoraux de l'Euripe (Achilléide, 449-450) mais sans précision supplémentaire. Quant à Callimaque, il précise que, de tous, ce sont les ports de l'Euripe qu'elle préfère (Hymn. Art., 188). Dans l'état actuel de nos connaissances la densité des sanctuaires du corpus se révèle déjà élevée. Il est donc temps d'ajouter à ces certitudes une conjecture. Elle concerne cette Aithopiè Braurônis dont je ne savais trop quoi faire il y a quelques années.

Une épigramme d'Antipater de Thessalonique ( $A P$, VII, 703) fait connaître le temple de la "brauronienne Aithopia» à Amphipolis. Pour rendre à cette divinité son arrière-plan cultuel, il fallait la rapprocher d'une Artémis Aithopia connue par une dédicace d'une femme à Mytilène $(I G$, XII 2, 92). G. Dunst (1959) a fait connaître une autre inscription d'Érythrées mentionnant le sacerdoce d'une Artémis Aithopia; texte repris et discuté par Fr. Graf (1987). Le témoignage le plus riche est celui d'une épigramme de l'époque classique ( $A P, \mathrm{VI}, 269)$, en dialecte éolien, destinée à la dédicace d'une statue féminine par Arista, propolos d'Artémis Aithopia, celle-ci étant dite fille de Léto et Despoina gynaikôn. Le texte s'adresse aux paides, qui pourraient bien être, comme le proposait G. Dunst, les propoloi de l'Aithopia. Il me semble légitime de restituer des rites de la jeunesse (pas seulement féminine peut-être) dédiés à une divinité qui, par ailleurs, comme la Brauronia de Brauron, est intimement attachée à la sphère féminine.

Si l'hypothèse de départ est exacte, Aithopia doit être aussi un nom de lieu. C'est le cas. Mais la question est embrouillée. Selon Pline c'est à Lesbos ( $H N, \mathrm{~V}, 139)$, selon Hésychius à Samothrace, selon Stéphane de Byzance (difficle à croire; cf. J. et L. Robert, Bull., 1960, 344) en Lydie, enfin selon Harpocration et la Souda en Eubée. La question se complique en raison de variations dans le nom : Aithopion ou Aithiopion. Quoi qu'il en soit, plusieurs sites peuvent évidemment porter le même nom, et il m'intéresse ici surtout que soit mise en évidence une zone où la fréquence des occurrences est grande : l'Ionie du nord et quelques places isolées : Amphipolis et l'Eubée. Le fait que ce soit l'excellent lexique de la langue des orateurs attiques qui glose 
Aithiopion "endroit de l'Eubée» invite à penser qu'une Artémis Aith(i)opia a pu y être vénérée. Il importe maintenant d'ajouter l'interprétation par Ératosthène de cette Aithopia, il l'assimile à Hécate «celle qui porte les torches" ( FGrH $241 \mathrm{~F} 46$ ). Les images associées de lumière et de chaleur conviennent tout à fait au sens de l'épiclèse. Or selon Hésiode et Stésichore, Artémis Hécate équivaut à Iphigénie (cf. Brulé, 1987, p. 267 n. 95). Cette Artémis Aith(i)opia est si proche de la Brauronia qu'elle reçoit l'hommage de la jeunesse, qu'elle est considérée comme despoina gunaikôn (AP, VI, 269) et surtout qu'elle est nommée «brauronienne» à Amphipolis ( $A P$, VII, 703). Si le sanctuaire euboïque d'Aithiopia entre bien dans la même série que ceux d'Amphipolis, de Mytilène et d'Érythrées, il devrait se trouver comme eux et comme ceux du corpus, proche des sources de catharsis : l'eau douce et l'eau salée. Or un détail mérite alors d'être rapproché : le sanctuaire de la Brauronia d'Amphipolis se trouve en bordure du Strymon, alors que l'Aulidéia d'Aulis se trouve sur les berges d'un autre Strymon, le sanctuaire de la Brauronia attique est traversé par l'Érasinos, alors que le même Érasinos traverse le site décrit par E. Sapouna-Sakellaraki comme étant celui de l'Amarysia. Il n'y a peutêtre pas là qu'une coïncidence.

Pierre BRULÉ

Université de Haute-Bretagne

Rennes 2

6 , avenue Gaston Berger

F - 35043 RENNES Cedex 
BRULÉ P., 1987 : La fille d'Athènes. Le religion des filles à Athènes à l'époque classique : mythes, cultes et société, Annales littéraires de l'Université de Besançon $\mathrm{n}^{\circ} 363$, Paris.

DUNST G., 1959 : Artemis Aithiopia. Zur erythräischen Inschrift über den Verkauf der Preistertümer, Philologische Vorträge Wroclaw, 85-87.

GRAF Fr., 1987 : Nordionische Kulte : Religiongeschichtliche und epigraphische Untersuchungen $z u$ den Kulten von Chios, Erythrai, Klazomenai und.Phokaia, Rome.

PALAIOKRASSA L., 1983 : Tò iє

RINGWOOD I.C., 1929 : Local Festivals of Euboea, AJA, 33, 385-92.

SALE W., 1975 : The Temple-Legends of the Arkteia, RhM, 118, 265-284.

SAPOUNA-SAKELLARAKI E., 1991 : Le sanctuaire d'Artémis Amarysia en Eubée, Kernos, 5, 235-263. 